\title{
Coupling between surface plasmons via thermal emission of a dielectric layer sandwiched between two metal periodic layers
}

\author{
Ming-Wei Tsai, Chia-Yi Chen, Yu-Wei Jiang, Yi-Han Ye, Hsu-Yu Chang, \\ Tzu-Hung Chuang, and Si-Chen Lee ${ }^{\text {a) }}$ \\ Department of Electrical Engineering, Graduate Institute of Electronics Engineering, National Taiwan \\ University, Taipei, 106, Taiwan, Republic of China
}

(Received 17 May 2007; accepted 16 August 2007; published online 20 November 2007)

\begin{abstract}
Metal $/ \mathrm{SiO}_{2} /$ metal trilayer thermally generated infrared emitters with different top and bottom periodic metal arrangements were fabricated and their emission spectra were measured. The coupling mechanisms of surface plasmon polaritons at top and bottom periodic metal structures were characterized. It is found that the top surface plasmon converted to light radiation directly, whereas the bottom surface plasmon can radiate out when its emission peak position matches that of the top surface plasmon. This opens the way to fabricate the high temperature operated, narrow bandwidth, and multiwavelength infrared light source. (C) 2007 American Institute of Physics. [DOI: 10.1063/1.2779964]
\end{abstract}

Infrared light source is valuable in the studies of the light induced reaction of biological system, environmental protection, industrial environment, and the defense industries. There are several methods to manufacture the infrared light source such as the quantum cascade lasers, ${ }^{1,2}$ photonic defect mode lasers ${ }^{3}$ and plasmonic thermal emitter. ${ }^{4-11}$ Threedimensional metallic photonic crystal with emission in the infrared region has been reported by Lin et al. ${ }^{6,7}$ The two dimensional narrow-band infrared thermal emitter was demonstrated by Pralle et al. ${ }^{4}$ Strong resonance of the air/Au surface plasmon polariton (SPP) degenerate modes resulted in the light radiation at the wavelength equal to the lattice constant. A two dimensional sandwiched $\mathrm{Ag} / \mathrm{SiO}_{2} / \mathrm{Ag}$ plasmonic thermal emitter was shown to exhibit strong $\mathrm{Ag} / \mathrm{SiO}_{2}$ and the Ag/air SPPs and converted to narrow bandwidth light radiation. ${ }^{8}$ Several theoretical studies based on plasmonic thermal emitter have been reported. ${ }^{9-11}$ Surface plasmons (SPs) are electromagnetic waves that occur at a metal/ dielectric interface with no light diffraction limitation. SPs result in a strongly enhanced nanoscale spatial distribution of an electric field near metal surface and can be used to the subwavelength photolithography. ${ }^{12}$ In this letter, the emission spectra of the $\mathrm{Ag} / \mathrm{SiO}_{2} / \mathrm{Ag}$ trilayer structure with different top and bottom periodic metal arrangements were measured. The coupling mechanisms of SPs at top and bottom metallic structures were characterized.

A Mo film was thermally deposited on the back of the double-polished Si substrate as a heating source. Ti and Ag metal films were deposited on the front side which can block the background radiation from the $\mathrm{Si}$ substrate. Then a $100 \mathrm{~nm} \mathrm{SiO}_{2}$ layer is deposited on top of the $\mathrm{Ag}$ reflective layer. After photolithography, the reactive ion etching was adopted to etch the $\mathrm{SiO}_{2}$ layer to the $\mathrm{Ag}$ reflective layer. Another $100 \mathrm{~nm} \mathrm{Ag}$ film was deposited to fill the etched area and lifted-off. The bottom Ag layer with periodic holes filled with $\mathrm{SiO}_{2}$ is formed. Then, a $100 \mathrm{~nm} \mathrm{SiO}$ layer was deposited as the sandwiched insulator. A photolithography step was again applied and the top periodic hole array of $100 \mathrm{~nm}$-thick silver film were evaporated and lifted-off. The top and bottom periodic structures of the trilayer

\footnotetext{
${ }^{a)}$ Electronic mail: sclee@cc.ee.ntu.edu.tw
}

$\mathrm{Ag} / \mathrm{SiO}_{2} / \mathrm{Ag}$ devices were listed in Table I. The schematic diagram showing the side view of the device structure was illustrated in Fig. 1(a). The radiation area of the sample is $1 \mathrm{~cm}^{2}$. A Perkin Elmer 2000 Fourier transform infrared spectrometer system was adopted to measure the thermal radiation spectra. The wave number resolution of the measurement is $8 \mathrm{~cm}^{-1}$. A dc was sent into the back Mo contact on $\mathrm{Si}$ substrate and heats up the sample lying in vacuum chamber.

The electromagnetic wave thermally generated in the $\mathrm{SiO}_{2}$ layer induced the SPs in the top and bottom metal films and then converted to light radiation. The bottom 200-nm-thick Ag can completely block the background radiation from the Si substrate.

The results of the similar sandwiched emitter but with only single periodic layer on top shown in Fig. 1(b) were presented elsewhere. ${ }^{8,13}$ It was shown that when the periodic structure was hexagonal arranged, only one specific peak was observed and attributed to $(1,0) \mathrm{Ag} / \mathrm{SiO}_{2}$ degenerate mode emission. If the periodic structure was square arranged, two emission peaks which corresponded to $(1,0) \mathrm{Ag} / \mathrm{SiO}_{2}$ and $(1,0) \mathrm{Ag}$ /air degenerate modes ${ }^{13}$ were observed. Table II lists the experimental peak positions of SP modes of a single periodic layer structure with different lattice structures (square and hexagonal) and lattice constants. The theoretical formula used to calculate the peak positions of SP modes are also given. The emission spectra were measured at the temperature of $240{ }^{\circ} \mathrm{C}$ for all devices. Figure 2(b) shows that the emission spectra of device $\mathrm{A}$, the peak at $4.16 \mu \mathrm{m}$ reshifted from the theoretical value of $3.6 \mu \mathrm{m}$, is composed of six degenerate modes, i.e., $( \pm 1,0),(0, \pm 1),(-1,1)$, and $(1,-1)$ $\mathrm{Ag} / \mathrm{SiO}_{2}$ modes designated as $(1,0)$ degenerate $\mathrm{Ag} / \mathrm{SiO}_{2}$

TABLE I. Periodic structure parameters for samples A, B, C, D, and E. The parameters of lattice constant, hole diameter, and side length are $a, d$, and $L$, respectively (unit: $\mu \mathrm{m}$ ).

\begin{tabular}{ccccccc}
\hline \hline Sample & Top & $a$ & $d$ or $L$ & Bottom & $a$ & $d$ or $L$ \\
\hline A & Hexagonal & 3 & 1.5 & Hexagonal & 3 & 1.5 \\
B & Hexagonal & 3 & 1.5 & Square & 5 & 2 \\
C & Square & 5 & 2 & Hexagonal & 3 & 1.5 \\
D & Square & 3 & 2 & Hexagonal & 5 & 2.5 \\
E & Hexagonal & 5 & 2.5 & Square & 3 & 2 \\
\hline \hline
\end{tabular}




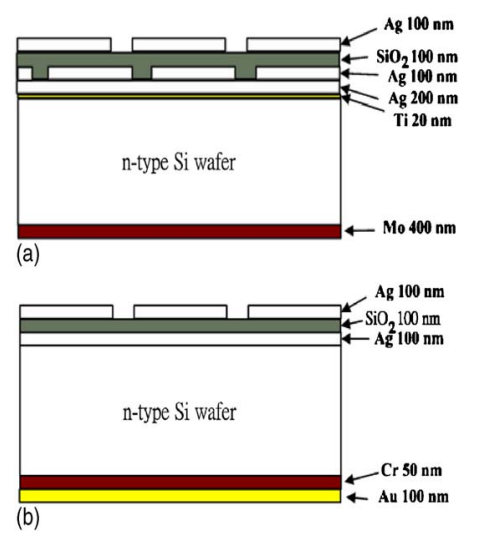

FIG. 1. (Color online) Schematic diagrams of the side view of the plasmonic thermal emitter. (a) Double layer device with both top and bottom metal films perforated with periodic hole array. (b) Single layer device with top metal film perforated with periodic hole array only.

mode. This is because the SP coupling between the top and bottom Ag films increases the effective dielectric constant of the sandwiched $\mathrm{SiO}_{2}$ layer and results in the redshift of the emission peak. ${ }^{14}$ According to Table II, the peak is generated from both top and bottom Ag layers and from a single periodic layer structure located at the same position shown in Fig. 2(a). When the bottom hexagonal lattice structure was replaced by the square lattice, device $\mathrm{B}$, the emission spectra were shown in Fig. 3(c). The peak at $4.49 \mu \mathrm{m}$ is the degenerate $(1,0) \mathrm{Ag} / \mathrm{SiO}_{2}$ hexagonal SP mode, but redshifts further from that of device A. By exchanging the top and bottom periodic metal structures, device C, as shown in Fig. 4(c), peaks at 3.53, 5.90, and $7.37 \mu \mathrm{m}$ were observed which were attributed to the bottom $(1,0) \mathrm{Ag} / \mathrm{SiO}_{2}$ hexagonal lattice $\mathrm{SP}$ mode without redshift and top $(1,0)$ Ag/air and $(1,0)$ $\mathrm{Ag} / \mathrm{SiO}_{2}$ square lattice $\mathrm{SP}$ modes, respectively. For device B, the emission spectra for single layer emitter [Fig. 1(b)] with the same structure as the bottom square and top hexagonal structures were shown in Figs. 3(a) and 3(b), respectively. The top $(1,0) \mathrm{Ag} / \mathrm{SiO}_{2}$ hexagonal mode is clearly seen in the combined structure, device $\mathrm{B}$, but the bottom $(1,0) \mathrm{Ag} / \mathrm{SiO}_{2}$ and $(1,0) \mathrm{Ag} /$ air modes disappear completely in the emission spectra because the top hexagonal lattice acts as a metallic filter which filtered out the thermal radiation from the bottom $\mathrm{Ag} / \mathrm{SiO}_{2}$ surface. In device $\mathrm{C}$, in addition to the two emission peaks coming from top $(1,0) \mathrm{Ag} / \mathrm{air}(\mathrm{A} / \mathrm{M})$ and $(1,0)$ $\mathrm{Ag} / \mathrm{SiO}_{2}(\mathrm{D} / \mathrm{M})$ square lattice modes [Fig. 4(b)], one of the bottom $(1,0) \mathrm{Ag} / \mathrm{SiO}_{2}$ hexagonal lattice $\mathrm{SP}$ mode [Fig. 4(a)] can also be seen in Fig. 4(c). This is because the bottom $(1,0)$ $\mathrm{Ag} / \mathrm{SiO}_{2}$ hexagonal lattice $\mathrm{SP}$ mode is coupled to the top $(2,0) \mathrm{Ag} / \mathrm{SiO}_{2}$ square lattice $\mathrm{SP}$ mode which results in the emission spectra of the hexagonal lattice. There is no align-

TABLE II. Measured emission peak positions of $(1,0)$ air/Ag and $(1,0)$ $\mathrm{SiO}_{2} / \mathrm{Ag}$ for single layer emitter with different lattice structures, lattice constant $a$, hole diameter $d$, and side length $L$ (unit: $\mu \mathrm{m}$ ). $\lambda=a\left(i^{2}+j^{2}\right)^{-1 / 2}\left(n_{d}\right)$ for square lattice and $\lambda=a\left(\frac{4}{3}\left(i^{2}+i j+j^{2}\right)\right)^{-1 / 2}\left(n_{d}\right)$ for hexagonal lattice. $i$ and $j$ are integers representing different surface plasmon modes. The refractive indices $n_{d}$ for air and $\mathrm{SiO}_{2}$ are 1 and 1.38 , respectively.

\begin{tabular}{ccccc}
\hline \hline Lattice type & $a$ & $d$ or $L$ & $(1,0) \mathrm{Ag} / \mathrm{air}$ & $(1,0) \mathrm{Ag} / \mathrm{SiO}_{2}$ \\
\hline Square & 3 & 2 & 3.3 & 5 \\
Square & 5 & 2 & 5.69 & 7.24 \\
Hexagonal & 3 & 1.5 & & 4.16 \\
Hexagonal & 5 & 2.5 & & 6.28 \\
\hline \hline
\end{tabular}

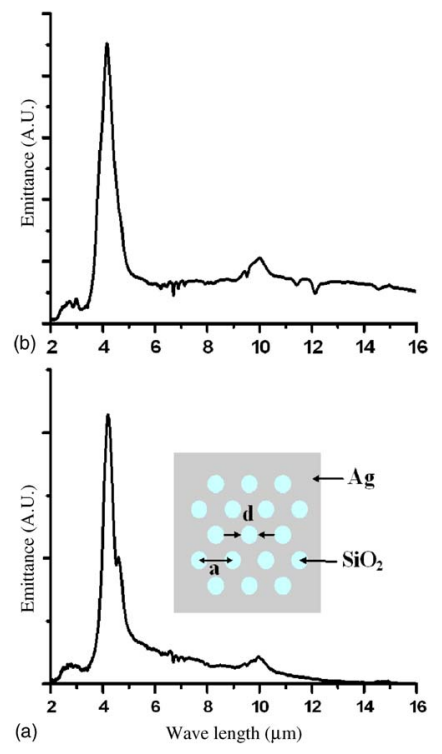

FIG. 2. (Color online) Measured emission spectra of a (a) single layer device with the same metal structure as the top or bottom periodic structure of device A, i.e., lattice constant $a=3 \mu \mathrm{m}$ and diameter $d=1.5 \mu \mathrm{m}$, and (b) double layer device A.

ment issue because the arrangement of the top and bottom periodic metal films is quite different.

In Fig. 5(c), the emission spectra of sample D with square lattice on top and hexagonal lattice at the bottom were shown. Two emission peaks at 3.62 and $5.11 \mu \mathrm{m}$ were attributed to the top $(1,0) \mathrm{Ag} / \mathrm{air}$ and $\mathrm{Ag} / \mathrm{SiO}_{2}$ square lattice $\mathrm{SP}$ modes which are similar to the single squared-lattice emission characteristics shown in Fig. 5(b). By exchanging the arrangement of the top and bottom periodic structures, device $\mathrm{E}$, the emission spectra were shown in Fig. 6(c). Peaks at $3.64,4.25$, and $6.97 \mu \mathrm{m}$ were attributed to $(1,0) \mathrm{Ag} / \mathrm{air}$ square, $(1,0) \mathrm{Ag} / \mathrm{SiO}_{2}$ square, and $(1,0) \mathrm{Ag} / \mathrm{SiO}_{2}$ hexagonal lattice SP modes, respectively. In device $\mathrm{D}$, the top $\mathrm{Ag} / \mathrm{SiO}_{2}$ and $\mathrm{Ag} /$ air square SP modes were clearly seen, but the bottom $\mathrm{Ag} / \mathrm{SiO}_{2}$ hexagonal mode shown in Fig. 5(a) disappears

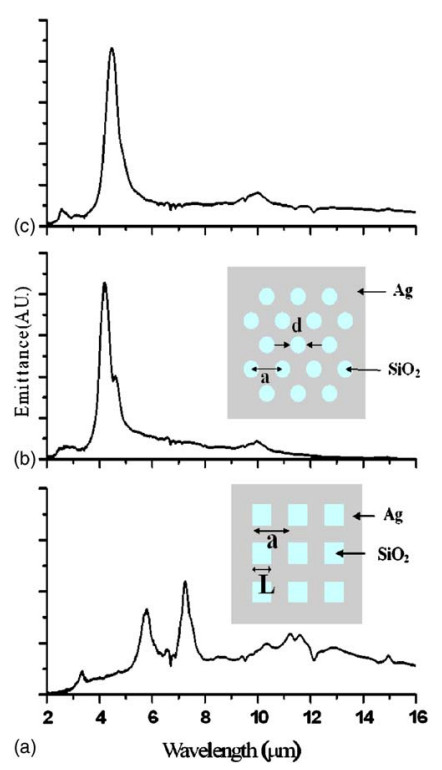

FIG. 3. (Color online) Measured emission spectra of a single layer device with the same metal structure as the (a) bottom periodic square structure of device B, i.e., lattice constant $a=5 \mu \mathrm{m}$ and length $L=2 \mu \mathrm{m}$. (b) Top periodic hexagonal structure of device B, i.e., lattice constant $a=3 \mu \mathrm{m}$ and diameter $d=1.5 \mu \mathrm{m}$. (c) Emission spectra of device B. 


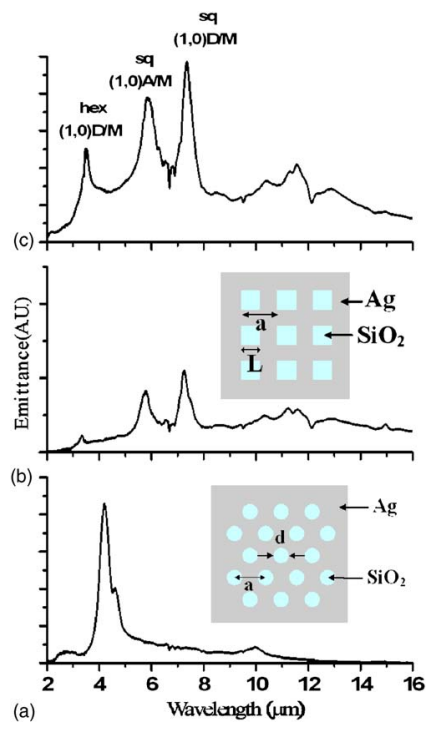

FIG. 4. (Color online) Measured emission spectra of a single layer device with the same metal structure as the (a) bottom periodic hexagonal structure of device C, i.e., lattice constant $a=3 \mu \mathrm{m}$ and diameter $d=1.5 \mu \mathrm{m}$. (b) Top periodic square structure of device C, i.e., lattice constant $a=5 \mu \mathrm{m}$ and length $L=2 \mu \mathrm{m}$. (c) Emission spectra of device C.

in the emission spectra. In device $\mathrm{E}$, the emission peaks come from the bottom $(1,0) \mathrm{Ag} /$ air square and $(1,0) \mathrm{Ag} / \mathrm{SiO}_{2}$ square lattice shown in Fig. 6(a) and the top $(1,0) \mathrm{Ag} / \mathrm{SiO}_{2}$ hexagonal SP modes shown in Fig. 6(b) were clearly seen. This is because the thermal radiation generated in $\mathrm{SiO}_{2}$ layer induced top and bottom SPs, the $(1,0) \mathrm{Ag}$ /air square SP mode is coupled to the top $(1,-1) \mathrm{Ag} / \mathrm{SiO}_{2}$ hexagonal SP mode, and the $(1,0) \mathrm{Ag} / \mathrm{SiO}_{2}$ square $\mathrm{SP}$ mode is coupled to the top $(1,0) \mathrm{Ag} /$ air hexagonal SP mode which results in the emission of bottom $\mathrm{Ag} / \mathrm{SiO}_{2}$ square $\mathrm{SP}$ mode.

Top SP converted to light radiation directly. The radiation route of bottom SP must penetrate through the top periodic Ag film. The key idea is that the top periodic structure acts as a filter. Whether the emission of the bottom layer can come out depends on the matching of the emission peak positions to those of the top periodic metal structure.

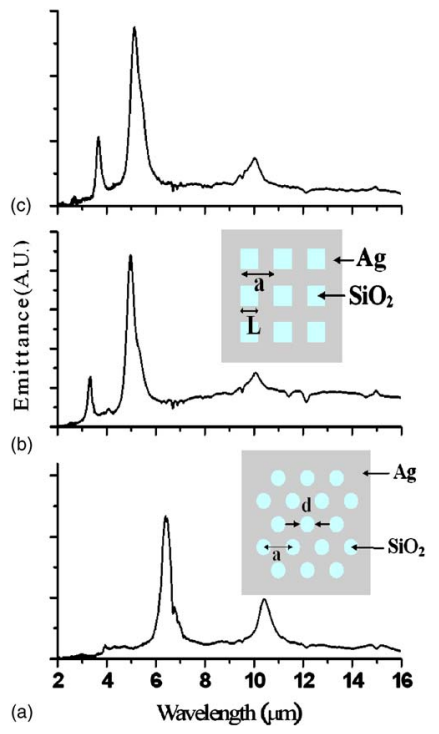

FIG. 5. (Color online) Measured emission spectra of a single layer device with the same metal structure as the (a) bottom periodic square structure of device D, i.e., lattice constant $a=3 \mu \mathrm{m}$ and length $L=2 \mu \mathrm{m}$. (b) Top periodic hexagonal structure with the lattice constant $a=5 \mu \mathrm{m}$ and diameter $d$ $=2.5 \mu \mathrm{m}$. (c) Emission spectra of device D.

Downloaded 04 Mar 2009 to 140.112.113.225. Redistribution subject to AIP license or copyright; see http://apl.aip.org/apl/copyright.jsp

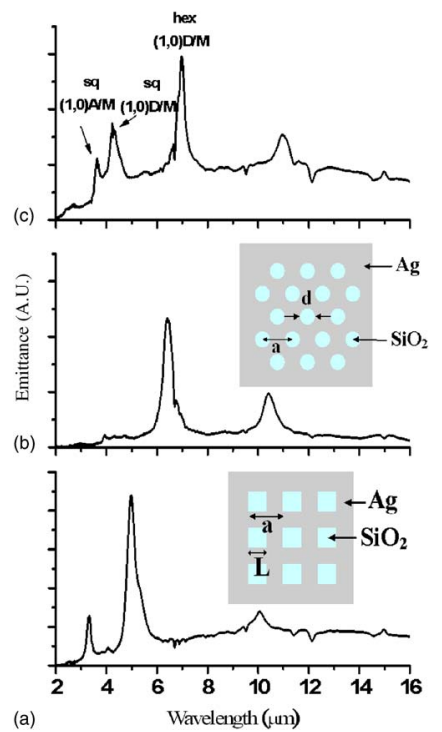

FIG. 6. (Color online) Measured emission spectra of a single layer device with the same metal structure as the (a) bottom periodic hexagonal structure of device E, i.e., lattice constant $a=5 \mu \mathrm{m}$ and diameter $d=2.5 \mu \mathrm{m}$. (b) Top periodic square structure with the lattice constant $a=3 \mu \mathrm{m}$ and length $L$ $=2 \mu \mathrm{m}$. (c) Emission spectra of device E.

In conclusion, $\mathrm{Ag} / \mathrm{SiO}_{2} / \mathrm{Ag}$ trilayer thermally generated infrared emitters were fabricated. The SP coupling mechanisms of top and bottom periodic metal structures were characterized. Top SP converted to light radiation directly. Whether the bottom SP can radiate out depends on its emission wavelength matching that of the top SP. It opens the way to fabricate various optoelectronic devices and can be used as a high temperature operated, narrow bandwidth, and multiwavelength infrared light source. This provides opportunities to investigate the biological system of the cells and plants as well.

The authors would like to thank the National Science Council of the Republic of China, Taiwan, for financially supporting this research under Contract No. NSC 95-2215E-002-042.

${ }^{1}$ Claire Gmachl, Deborah L. Sivco, Raffaele Colombelli, Federico Capasso, and Alfred Y. Cho, Nature (London) 415, 883 (2002).

${ }^{2}$ Richard Maulini, Mattias Beck, Jérôme Faist, and Emilio Gini, Appl. Phys. Lett. 84, 1659 (2004).

${ }^{3}$ Hong-Gyu Park, Se-Heon Kim, Soon-Hong Kwon, Young-Gu Ju, Jin-Kyu Yang, Jong-Hwa Baek, Sung-Bock Kim, and Yong-Hee Lee, Science 305, 1444 (2004).

${ }^{4}$ M. U. Pralle, N. Moelders, M. P. McNeal, I. Puscasu, A. C. Greenwald, J. T. Daly, E. A. Johnson, T. George, D. S. Choi, I. El-Kady, and R. Biswas, Appl. Phys. Lett. 81, 4685 (2002).

${ }^{5}$ Irina Puscasu, M. Pralle, M. McNeal, J. Daly, A. Greenwald, E. Johnson, R. Biswas, and C. G. Ding, J. Appl. Phys. 98, 013531 (2005).

${ }^{6}$ S. Y. Lin, J. Moreno, and J. G. Fleming, Appl. Phys. Lett. 83, 380 (2003).

${ }^{7}$ Shawn-Yu Lin, J. G. Fleming, and I. El-Kady, Appl. Phys. Lett. 83, 593 (2003).

${ }^{8}$ Ming-Wei Tsai, Tzu-Hung Chuang, Chao-Yu Meng, Yi-Tsung Chang, and Si-Chen Lee, Appl. Phys. Lett. 89, 173116 (2006).

${ }^{9}$ David L. C. Chan, Marin Soljačić, and J. D. Joannopoulos, Opt. Express 14, 8785 (2006)

${ }^{10}$ R. Biswas, C. G. Ding, I. Puscasu, M. Pralle, M. McNeal, J. Daly, A. Greenwald, and E. Johnson, Phys. Rev. B 74, 045107 (2006).

${ }^{11}$ David L. C. Chan, Marin Soljačić, and J. D. Joannopoulos, Phys. Rev. E 74, 016609 (2006).

${ }^{12}$ Zhao-Wei Liu, Qi-Huo Wei, and Xiang Zhang, Nano Lett. 5, 957 (2005).

${ }^{13}$ Tzu-Hung Chuang, Ming-Wei Tsai, Yi-Tsung Chang, and Si-Chen Lee, Appl. Phys. Lett. 89, 173128 (2006).

${ }^{14}$ T. Thio, K. M. Pellerin, R. A. Linke, H. J. Lezec, and T. W. Ebbesen, Opt. Lett. 26, 1972 (2001). 